\title{
Review
}

\section{Exposure to Ethylating Agents: Where Do the Thresholds for Mutagenic/Clastogenic Effects Arise?}

\author{
Elmar Gocke, Leilei Tang ${ }^{1}$ and Thomas Singer \\ Preclinical Research, F. Hoffmann-La Roche Ltd, Basel, Switzerland
}

(Received March 30, 2012; Revised July 2, 2012; Accepted July 6, 2012)

The dose response relationships for induction of micronuclei in bone marrow and for induction of lac $Z$ mutations in liver, large intestine, and bone marrow were assessed in mice after treatment with ethyl methanesulfonate (EMS). The animals were treated orally at doses between 1.25 to $260 \mathrm{mg} / \mathrm{kg}$ for up to 28 days. Statistical analysis indicated that the dose response curves were well compatible with a thresholded relationship with apparent thresholds $\geq 25 \mathrm{mg} / \mathrm{kg} /$ day. In contrast, no threshold was apparent for the induction of alkylation-adducts in proteins and DNA. An approximately linear dose dependency was observed, indicating that the macromolecular targets were alkylated proportionally to the given dose. It is concluded that the cells have the capacity to repair large amounts of EMS-induced alkylations, up to the threshold dose, virtually error free (i.e., without adding to the background burden of clastogenic/mutagenic events). Since the statistical power to define the 'true' shape of the dose response curve is much more limited for in vivo studies compared to in vitro experiments with e.g., a bacterial reverse mutation system we assessed the dose response relationship for EMS induced mutations at the his $\mathbf{G 4 6}$ locus in alkylation repair proficient (TA1535, ogt ${ }^{+}$) and deficient (YG7104, (logt) bacteria using as many as 23 dose levels. Clear sublinearity was apparent for TA1535 while linearity was obvious for YG7104. Applying curve fitting with a 'hockeystick' model a threshold dose of about $750 \mu \mathrm{g} / \mathrm{plate}$ was determined for TA1535. With curve fitting using a benchmark model (PROAST) we estimated the efficiency of error-free repair by the ogt system at the different EMS exposures. The likelihood of erroneous repair approaches 0 with decreasing EMS concentrations. Together with recent studies on other alkylating agents our data argue for a change of paradigm concerning risk assessment of the exposure to simple DNA-alkylating genotoxins.

Key words: dose response, threshold, ethyl methanesulfonate, alkylating agents, risk assessment

\section{Introduction}

Mutations and/or clastogenic effects generally arise from exposure to a genotoxic (DNA-damaging) agent. The mutagenic/clastogenic alteration might occur directly (i.e., by mispairing); or might be related to er- roneous or unsuccessful/incomplete repair of the induced DNA lesion. Traditionally, the dose-response relation for mutagenic effects has been thought to have no threshold; that is, even the most miniscule exposure carries a finite (non-zero) chance for eliciting the effect. Recently, several publications have provided convincing experimental evidence that simple alkylating agents, such as methyl methanesulfonate (MMS) and ethyl methanesulfonate (EMS), cause mutational events with a thresholded dose-response relationship (1-3).

When considering possible mechanisms for a thresholded dose-response relationship, two distinctive possibilities, both involving saturation processes, come to mind:

1) a thresholded dose response can arise if a scavenging and/or detoxification mechanism fully prevents accessibility of the genotoxin to the DNA (and thus averts induction of damages) up to a certain dose level;

2) a thresholded dose response can arise if a repair mechanism is capable of removing all DNA damages practically error free up to a certain damage level-even if DNA damages are induced in dose proportional fashion.

We have provided evidence that the latter mechanism is responsible for thresholds in the dose response of the induction of micronuclei and gene mutations in mice treated daily for 7 or 28 days with EMS. In the present article, we further augment our arguments by describing dose response curves for mutation induction in repair proficient and deficient bacteria. We show that chance for erroneous repair in the ogt-repair proficient cells appears to approach zero with decreasing concentration of EMS resulting in a (practical) thresholded of the dose response curve.

Our in vivo studies on EMS were triggered by the need to assess the possible mutagenic/carcinogenic risk

${ }^{1}$ Correspondence to: Leilei Tang, Preclinical Research, F. HoffmannLa Roche Ltd, CH 4070 Basel, Switzerland. E-mail: leilei.tang@ roche.com

doi: $10.3123 /$ jemsge. 34.171 
of patients accidentally exposed to EMS after ingesting contaminated Viracept (Nelfinavir mesylate) tablets for a period of up to three months (4-8). EMS has long been known as a standard alkylating agent and is used as a positive control in many in vitro and in vivo genetic toxicity assays. Yet, existing in vitro and in vivo data did not permit a sufficiently convincing risk assessment for the levels at which patients were exposed to EMS (maximal dose of $0.055 \mathrm{mg} / \mathrm{kg} /$ day). Therefore, the marketing authorisation holder of Viracept, F. Hoffmann-La Roche, conducted a series of non-clinical investigations $(2,6,7,9)$ to better quantify the risk for adverse effects in the exposed individuals. For direct acting, DNA-damaging genotoxins, such as EMS, it has been generally assumed that dose-response relations are linear-or at least non-thresholded-due to their stochastic, all-ornothing, mode of action (10). By default, the formation of tumours, heritable birth defects, and teratogenic effects are also accepted to follow a linear dose relation for these genotoxins. However, the publication by Doak et al. (1) provided strong evidence for a thresholded dose-response relationship for in vitro induction of micronuclei and gene mutations by EMS. Based on this publication, our studies were aimed at obtaining reassuring evidence for the absence of (geno)toxicological adverse effects in the Viracept patients. Our studies provided strong evidence for a thresholded dose response regarding both clastogenic and mutagenic activities of EMS (11). The threshold dose was observed to be $\geq 25$ $\mathrm{mg} / \mathrm{kg} /$ day, orders of magnitude higher than the maximal patient dose $(2,11)$. This observation also held true $(7,8)$ when the assessment was based on drug metabolism and pharmacokinetics parameters (AUC, $\left.\mathrm{C}_{\max }\right)$. On this basis, it was concluded that the Viracept patients did not experience any additional toxicological adverse effects, which would be attributable to their exposure to EMS (8). This argument was accepted by the authorities and the initially issued request for registries for birth defects and/or tumorigenic effects in putatively affected patients was retracted (4).

In the present publication, we revisit the earlier studies focussing on the dose-response curves of protein and DNA adduct formation in comparison to the doseresponse curves of the clastogenic and mutagenic effects in the bone marrow of mice exposed to EMS. We further enhance on our argument that the thresholded dose response relations are due to error free repair by detailed analysis of the dose response curves for mutation induction in ogt-repair proficient and deficient strains of $S$. typhimurium. Bacterial studies on the model mutagen EMS have been published in the literature in large numbers. The type of DNA damages induced and their reversal and repair by a multitude of complex pathways have been described in great detail (12-19). Here we concentrate on curve fitting and mathematical model- ling of the dose response curves rather than on the discussion of detailed molecular processes.

\section{Material and Methods}

The experimental details of the MNT and $\mathrm{Muta}^{\mathrm{TM}}$ Mouse studies are described in Gocke et al. (2). The genotoxicity tests were performed under GLP at Covance, Harrogate, UK. Formulation analytics of EMS were conducted at F. Hoffmann-La Roche Ltd, Basel (for MNT test) and RCC Ltd, Itingen, Switzerland (for Muta ${ }^{\mathrm{TM}}$ Mouse test). Bioanalytics (determination of ethylvaline adduct levels) was performed at Currenta GmbH \& Co, Leverkusen, Germany.

Test item: EMS [CAS 62-50-0] was purchased from Sigma-Aldrich, UK. Aqueous solutions of the appropriate concentrations were administered by oral gavage.

Micronucleus (MNT) study: The protocol of the MNT was designed to meet the known requirements of the OECD Guideline 474, 1997 and the ICH Tripartite Harmonised Guideline on Genotoxicity: Specific Aspects of Regulatory Tests, 1995 (2). Outbred Crl:CD1 (ICR) mice were obtained from Charles River (UK) Ltd, Margate, UK. Animals were treated daily for 7 days $(0,1.25,2.5,5,20,80,140,200,260 \mathrm{mg} / \mathrm{kg} /$ day $)$ and necropsied on day 8. Four thousand PCE per animal were scored for MN (MN-PCE) from 6 animals per dose point.

Muta ${ }^{\mathrm{TM}}$ Mouse study: The Muta $^{\mathrm{TM}}$ Mouse study was designed to comply with the recommendations made at the IWGT meeting in 2002 (2). $\mathrm{CD}_{2}$-lacZ80/HazfBR mice were obtained from Harlan, UK. The study consisted of two arms: a 28-day, daily treatment scheme (EMS: 0, 1.56, 3.13, 6.25, 12.5, 25, 50, $100 \mathrm{mg} / \mathrm{kg} /$ day, sampling on day 31) and a single treatment scheme (EMS: $0,350 \mathrm{mg} / \mathrm{kg}$, sampling on day 7 or day 31 ). Where possible, data were generated for at least 200,000 pfu per tissue for all 7 animals per group.

Protein adduct determination: Blood was obtained from each animal during necropsy by cardiac puncture and processed for analysis of ethylvaline levels. The determinations were made for all animals treated with EMS $(2,6)$.

Bacterial reverse mutation (Ames test): The standard procedure for the Ames plate incorporation test was followed (20). In addition to the standard tester strain TA1535 $\left(\mathrm{ogt}^{+}\right)$the repair deficient derivative YG7104 ( $\Delta$ ogt), kindly supplied by T. Nohmi, was employed (16). 23 dose points (three plates/dose) spaced evenly by a factor of 1.22 were used in addition to 21 solvent control plates.

Statistical assessment: The statistical assessment of the in vivo genotoxicity data is described in detail in Gocke and Wall (11). The threshold software developed by Lutz and Lutz (21) was employed to calculate the threshold dose values including confidence limits. For 
assessing the dose response of the bacterial tests the PROAST software developed by Slob et al. (22) was employed in addition to the Lutz and Lutz 'hockeystick' model.

\section{Results \\ In Vivo Studies with EMS}

In the course of our studies (2) on the genotoxic activity of EMS, we investigated its clastogenic action in bone marrow and its mutagenic action in bone marrow, liver and large intestine.

In these studies, the induction of globin adducts in peripheral blood was also investigated as a measure of exposure in order to model pharmacokinetics (7) in the patients exposed to EMS-contaminated Viracept. In Fig. 1 the genotoxic effects and the adduct levels are plotted relative to their corresponding control values. It is apparent that low dose levels do not result in increases of the mutagenic/clastogenic effects but they do substantially increase the level of adducts. Indeed, for the clastogenicity endpoint, even a negative (hormesis-like) trend was apparent, which was assessed as being statistically significant (11). Above the NOEL doses of 25 to 50 $\mathrm{mg} / \mathrm{kg} /$ day for lacZ induction and $80 \mathrm{mg} / \mathrm{kg} /$ day for $\mathrm{MN}$ induction, clear increases of the genotoxic effects are observed, reaching a factor of $8.7(\mathrm{MN})$ at dose 200 $\mathrm{mg} / \mathrm{kg} /$ day or 4.0 (lacZ) at dose $100 \mathrm{mg} / \mathrm{kg} /$ day relative to the respective control values. Using the threshold software developed by Lutz and Lutz (21), we obtained estimates of the threshold values and confidence intervals. Figure 2 shows a representative 'hockeystickmodelling' for lacZ mutations in bone marrow. Table 1 summarizes the data for all endpoints.

In contrast to the clastogenic and mutagenic effects, it

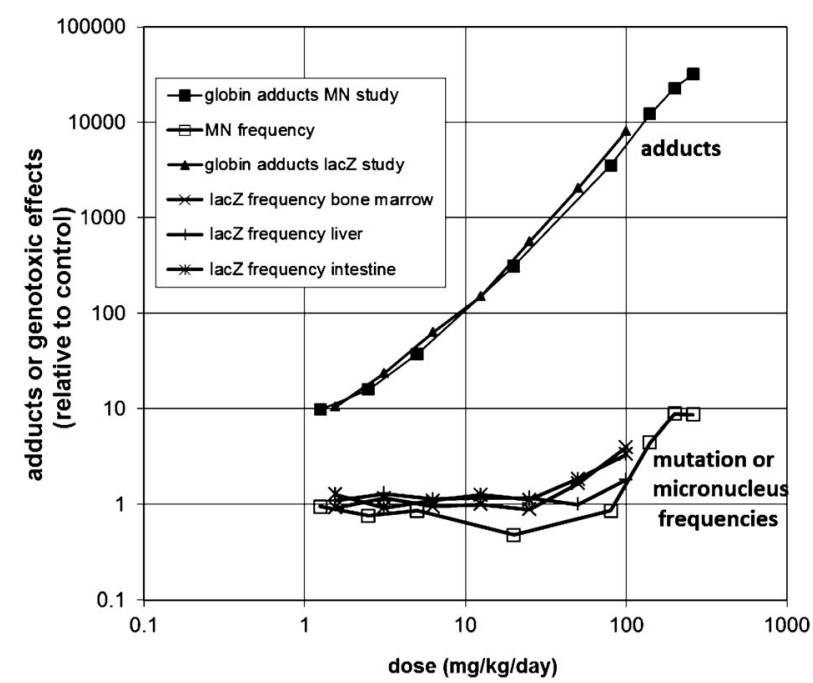

Fig. 1. Induction of protein adducts in globin, micronuclei and $l a c Z$ mutations in bone marrow. All values plotted relative to the respective control values. is evident that no threshold for adduct formation can be derived from the data shown in Fig. 1. In fact, an almost 10 fold increase over background is apparent already at the lowest dose of about $1 \mathrm{mg} / \mathrm{kg} /$ day. At the threshold doses for genotoxicity, the ethylvaline levels surpass the background values by a factor of about 1000 .

In our studies $(2,6)$ we determined globin adducts as a measure of exposure in order to model pharmacokinetics of EMS in patients (7). Previous investigations of Murthy et al. (23) determined adduct levels in DNA. Comparing these data to our results showed a high degree of parallelity for ethylation of both targets as depicted in Fig. 3. Murthy et al. (23) used radioactive EMS, enabling them to obtain reliable data down to dose levels below $0.1 \mathrm{mg} / \mathrm{kg}$ of EMS because background ethylation does not interfere with the measurements. Figure 3 shows, at doses below $10 \mathrm{mg} / \mathrm{kg}$, they observed a roughly linear dose relation (i.e., slopes in the log-log plot of about 1), and, at doses above 10

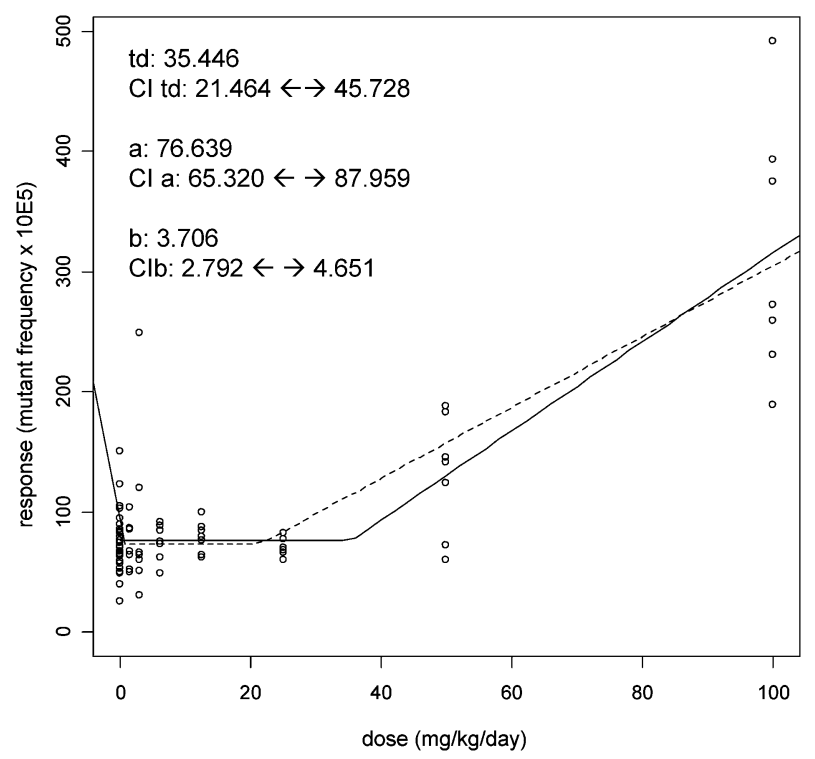

Fig. 2. Threshold analysis for the bone marrow Muta ${ }^{\mathrm{TM}}$ Mouse data with Lutz and Lutz hockeystick software (14). The inserted values give the values and $95 \%$ Confidence Interval of td (threshold), a (spontaneous mutation frequency) and $b$ (slope at doses $>t d$ ).

Table 1. Threshold analysis overview $(11,21)$

\begin{tabular}{llccc}
\hline \hline \multicolumn{1}{c}{ Study } & \multicolumn{1}{c}{ Organ } & $\begin{array}{c}\text { NOEL } \\
(\mathrm{mg} / \mathrm{kg})\end{array}$ & $\begin{array}{c}\text { Threshold } \\
(\mathrm{td}) \\
(\mathrm{mg} / \mathrm{kg})\end{array}$ & $\begin{array}{c}95 \% \\
\text { Confidence } \\
\text { interval of td } \\
(\mathrm{mg} / \mathrm{kg})\end{array}$ \\
\hline MNT & Bone marrow & 80 & 89.8 & 56.7 to 118.2 \\
Muta ${ }^{\mathrm{TM} M o u s e}$ & Bone marrow & 25 & 35.4 & 21.5 to 45.7 \\
Muta $^{\mathrm{TM} M}$ Mouse & Liver & 50 & 51.3 & 25.7 to 100.0 \\
Muta $^{\mathrm{TM}}$ Mouse & Large Intestine & 25 & 24.5 & 13.0 to 38.5 \\
\hline
\end{tabular}




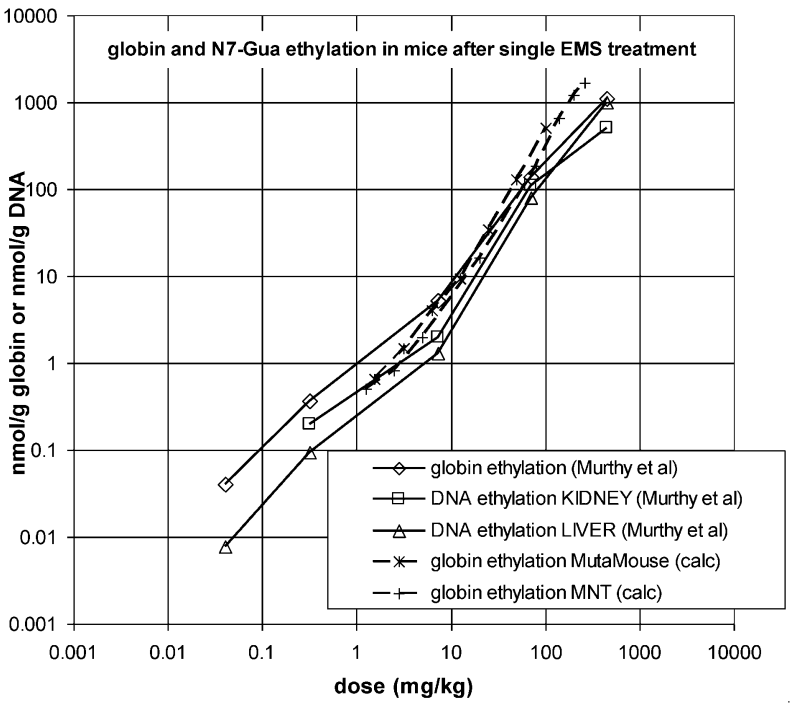

Fig. 3. Adduct levels in DNA and globin after single doses of EMS. Note: Data are taken from the study by Murthy et al. (16) with radioactive EMS or are calculated from our MN and Muta ${ }^{\mathrm{TM}}$ Mouse studies using the formula for calculating the daily increment (17) and the relative reactivity with ethylvaline versus total globin determined by Murthy et al. in whole blood (16).

$\mathrm{mg} / \mathrm{kg}$, they observed a nearly quadratic relation (i.e., slope close to 2 in log-log plot). When calculating the daily increments of globin- adduct levels in our 7 or 28 day studies (2), our values compare well with the data reported by Murthy et al. (23).

\section{In Vitro Study with EMS in S. typimurium}

In order to define more closely the impact of DNA repair on the dose response of mutation induction we decided to use the S. typhimurium system with strain TA1535, which is alkyltransferase proficient, and its derivative YG7104 ( $\Delta$ ogt) which is deficient for the ogt methyltransferase system. The ogt system was previously shown to constitute the main repair pathway in S. typhimurium for ethylating agents like EMS (17). Investigations on the dose response of EMS in various bacterial systems have been performed many years ago and are summarized by Matijasevic and Zeiger (24).

It is immediately apparent that TA1535 is less sensitive to EMS mutagenicity by orders of magnitude. Not only the slope but also the shape of the dose response curves differs between the two strains. The revertant frequency curve of TA1535 shows a clear sublinearity while for YG7104 a linear increase is apparent at the low dose range (at higher doses the downward curvature is due to toxicity and lower counting efficiency of the image analysis system at high colony densities). Consequently, the Lutz and Lutz software does not reject linearity and does not identify a threshold for YG7104 (data not shown) but returns a value of $773 \mu \mathrm{g} /$ plate as threshold dose for EMS in TA1535 (Fig. 5). It is noted

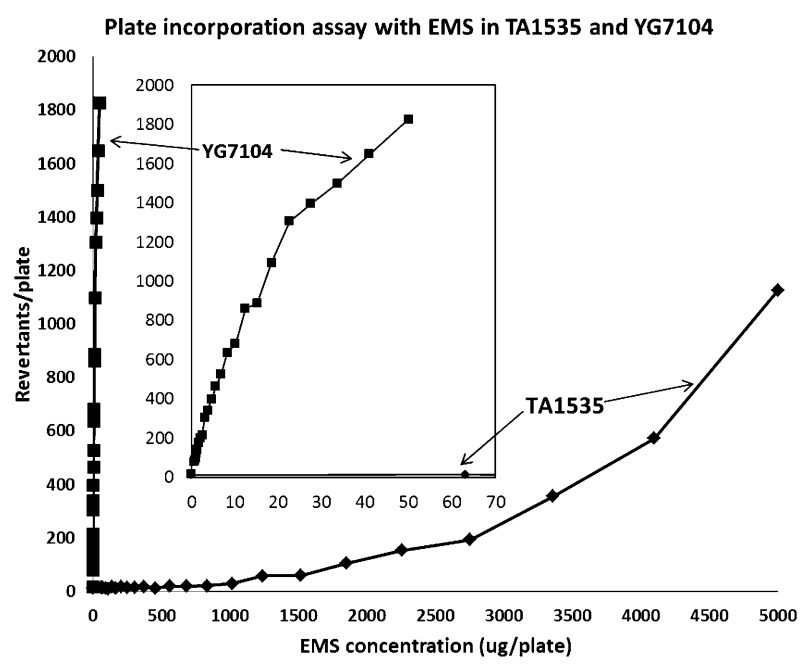

Fig. 4. Bacterial reverse mutation test with EMS. Insert: Magnification of low dose range.

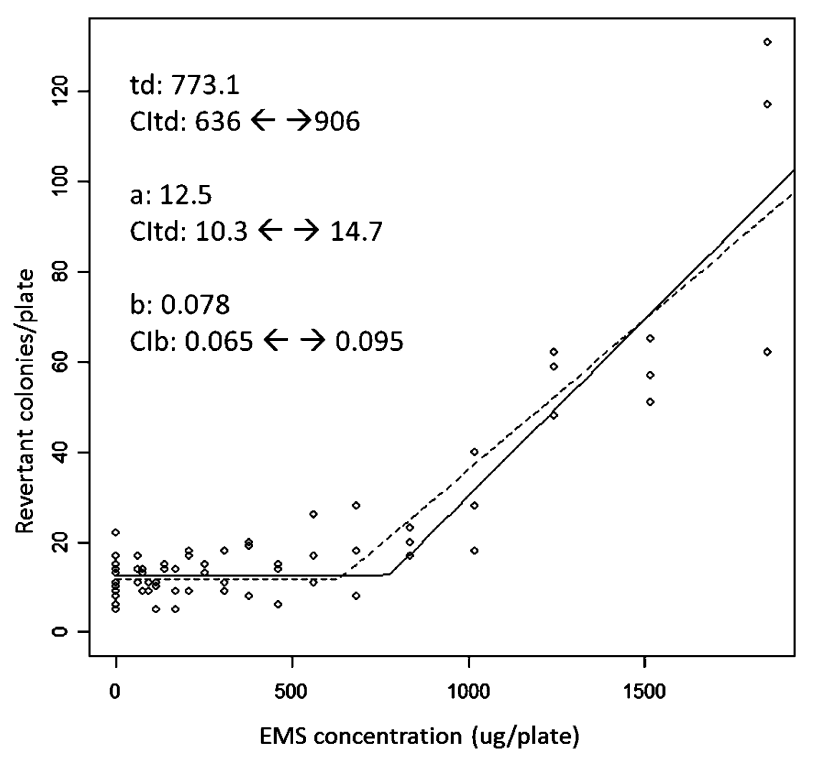

Fig. 5. Threshold analysis of the bacterial reverse mutation data with Lutz and Lutz hockeystick software. The inserted values give the values and $95 \%$ Confidence Interval of td (threshold), a (spontaneous revertants) and $\mathrm{b}$ (slope at concentrations $>\mathrm{td}$ ).

that due to the continuously increasing upward curvature the application of the hockeystick model is not appropriate for the whole dose range as it would result in an obvious overestimate of the td value (not shown). Therefore we limited the evaluated dose range in Fig. 5 to concentrations of up to $2500 \mu \mathrm{g} /$ plate where the increase of the revertant frequency was not more than 10 fold over control.

In an alternative approach we fitted the data with the PROAST software (22) using the exponential model

$$
y=a *\left(c-(c-1) * \exp \left(-b * x^{d}\right)\right.
$$




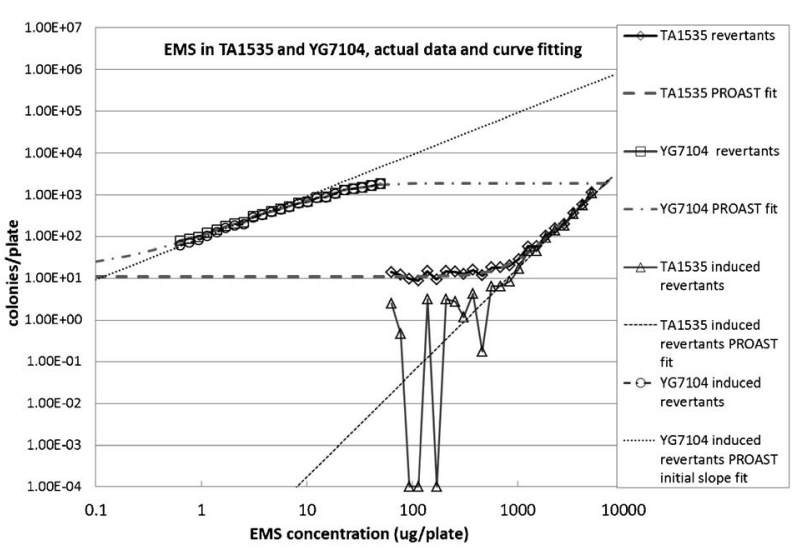

Fig. 6. Analysis of dose response relations for the induction of mutations in S. typhimurium strains. Data of strains TA1535 and YG7104 are fitted with the PROAST software (15).

Note: For YG7104 the leveling off of the number of revertant colonies/plate at higher ( $>10 \mu \mathrm{g} /$ plate) concentrations is mainly due to inefficient colony separation at high colony densities and start of toxicity. The extrapolation of the linear fit in the low dose range (with the initial slope calculated in the text for YG7104) can be seen as representing the number of pro-mutagenic lesions induced in the absence of the ogt system. For induced revertants of TA1535 negative values (i.e., where colony number on treated plate was lower than on control plates) a value of $1^{*} 10^{-4}$ was inserted as negative values cannot be plotted in log-log scale.

The constants a, b, c, d define background level, potency ('rapidity' of increase), maximum response, and shape of the curve (15). Fitting the TA1535 and YG7104 data resulted in the following equations $(\mathrm{r} f=$ revertant frequency, $\mathrm{x}=$ concentration of EMS in $\mu \mathrm{g} /$ plate)

$$
\begin{aligned}
r f_{1535}= & 11 *(1000-(1000-1) * \exp \\
& \left.\times\left(-4.995 * 10^{-11} * x^{2.5}\right)\right)
\end{aligned}
$$

and

$$
r f_{7104}=15.9 *(118-(118-1) * \exp (-0.04915 * x))
$$

In Fig. 6 we show the frequency of revertant colonies, induced revertant colonies and the fitted curves in a log$\log$ plot. We estimate the repair efficiency by the ogt system as a function of the EMS concentration, as discussed below.

The PROAST software calculates the concentration of $252 \mu \mathrm{g} /$ plate as benchmark dose (= estimate of dose increasing the colony numbers by $5 \%$ ) for TA 1535 while a roughly 30,000 fold lower benchmark dose $(0.0087$ $\mathrm{ug} /$ plate) is calculated for YG7104. It is notable that the fitting resulted in $d=1$ for YG7104 and $d=2.5$ for TA1535. This has decisive implications for the slope (i.e., the first derivative):

$$
y^{\prime}=a *(c-1) * b * x^{d-1} * \exp \left(-b * x^{d}\right)
$$

At low concentrations the slope for YG7104 (with $d=1$ ) approaches the value of 91.4

$$
{ }_{x \rightarrow 0} \mathrm{rf}_{7104}^{\prime} \rightarrow 91.4 \text { revertants/ug of EMS }
$$

and for TA1535 (with $\mathrm{d}=2.5$ ) the slope approaches 0

$$
x \rightarrow 0 \mathrm{rf}_{1535}^{\prime} \rightarrow 0 \text { revertants/ug of EMS. }
$$

Consequently, this analysis agrees the assessment with the Lutz and Lutz (21) 'hockeystick model' that a linear dose relationship is seen for YG7104 and a threshold can be seen for TA1535.

\section{Discussion}

EMS has been investigated in numerous in vitro and in vivo studies for the induction of gene mutations and clastogenic events $(1,12,24,26)$. In vivo rodent studies employing doses of $\geq 50 \mathrm{mg} / \mathrm{kg} /$ day were not amenable to the elucidation of dose-response relationships at lower doses, which was of particular relevance for assessing the genotoxic risk of the patients exposed to EMS-contaminated Viracept (4). Graphical assessment of these published in vivo studies, as presented in Gocke et al. (26), suggested sub-linear dose relations for almost all investigations. However, definite conclusions from these studies could not be drawn because of experimental restrictions (e.g., lack of doses below the LOEL, too few animals in controls and treatment groups, and too few cells analysed per animal).

Our studies (2) were designed to give a reliable experimental and statistical basis for determination of a threshold dose, should it exist in vivo. Thus, we employed 7 to 8 closely spaced dose levels starting as low as $1.25 \mathrm{mg} / \mathrm{kg} / \mathrm{day}$, included several (up to 4) independent control groups, increased the number of animals/dose group and the number of evaluated cells/animal above the levels recommended in the respective guidelines. This approach provided 5 to 6 dose levels that were identified to be below the LOEL dose.

Fixation of mutations likely depends on the status of proliferation and differentiation of the exposed cells. Studies with short-term treatments indicated that the sensitivity of stems cells might be higher or lower than the sensitivity of transit or differentiated cells, depending on the organ investigated (27). In our Muta ${ }^{\mathrm{TM}}$ Mouse study, we included three organs (liver, intestine, bone marrow) with different turnover rates, and the treatment period of the study was chosen to be 28 days, so that the induction of lacZ mutations was measured in differentiated, transit and stem cells in the different organs. We also included a single, acute treatment regiment to show that dose fractionation abolishes the mutagenic effect when the daily dose is below the threshold, while the acute dose, equal to the total cumulative dose, induces a clear increase (2).

We believe our data provide unambiguous and reliable support for a thresholded dose-response relationship. When applied to human risk assessment, the 'traditional' approach of linear back-extrapolation for DNA damaging genotoxins/carcinogens can be replaced 
by the application of appropriate safety factors (8) as has already been accepted for non-DNA damaging genotoxins.

While the dose response relations for genotoxic effects were assessed to follow a 'hockey-stick' fashion, our data on globin ethylation, as well as the data of Murthy et al. (23) on the alkylation of N7-G ethylation, provide convincing evidence that induction of alkylation damage after EMS exposure does not occur in a threshold manner down to dose levels of $0.1 \mathrm{mg} / \mathrm{kg}$ (Figs. 1, 3). This implies that there is no efficient scavenging of EMS prior to reaction with DNA. As a consequence, the appearance of thresholds in the doseresponse curve for clastogenic and mutagenic effects has to be attributed to error-free removal of DNA damage. In a calculation described in Gocke and Müller (28), we have derived that in liver cells treated at the NOEL dose of lacZ mutation induction $(50 \mathrm{mg} / \mathrm{kg} /$ day $)$ each daily EMS dose induced 380,000 DNA alkylations without any measurable increase in mutation frequency. For bone marrow cells, the calculation yields a total of 78,000 adducts at the NOEL of $25 \mathrm{mg} / \mathrm{kg} /$ day, assuming a similar adduct induction in bone marrow DNA as in liver DNA. The different adduct levels at the threshold doses in the two organs might be due to the different cell turnover (i.e., the liver cells have more time to repair) or could be attributed to different capacities of the repair systems in the two organs (29).

Zair et al. $(30,31)$ have recently shown that the threshold for induction of micronuclei in vitro is critically impacted by the N-methylpurine DNA glycosylase (MPG)/base excision (BER) repair system known to play a pivotal role in $\mathrm{N}^{7}$-ethyl Guanine repair. Complete ablation of a clastogenic threshold dose-response was seen in MPG-deficient human lymphoblastoid cells. Numerous studies on impact of repair capacities on shape of dose response have been published for EMS in the more distant history of mutagenesis research, as reviewed by Matijasevic and Zeiger (24). While induction of alkylations rises linearly with concentrations, the induction of mutations varied with the 2.13 and 4.20 power of exposure for $\mathrm{uvr}^{+}$and uvrB strains, respectively. Surprisingly, the excision-repair deficient strain showed relatively higher mutation frequencies at low concentrations but was less mutagenized at higher concentrations than the uvrB strains.

$\mathrm{O}^{6}$-Alkyl guanine is thought to be the most critical DNA lesion for induction of gene mutations because of its mispairing potential $(1,18,19)$. Direct removal of the adducts is provided by the alkyl transferase enzymes (13-17). In order to investigate the impact of the alkyltransferase enzymes on mutation induction we turned to the $S$. typhimurium system where repair deficient strains ( $\Delta$ ogt, $\Delta$ ada) derivatives of the standard tester strain TA1535 have been generated by the group of T. Nohmi (17). Detailed knowledge about DNA damage reversal by alkyltransferases, as well as about other repair systems for alkylation damage, has been accumulated in a multitude of studies $(13-19,29,30,32)$. For our intention to define dose response relations we specifically wanted to compare the effects of the main alkylation repair system on the shape of the dose response curves in a system where the impact of statistical variation can be minimized. The bacterial $S$. typhimurium histidin reversion system seemed well suited. As shown in Fig. 4, the revertant frequency increases linearly in strain YG7104 at low concentrations (up to about $8 \mu \mathrm{g} /$ plate), while the curve for TA1535 shows a very pronounced sublinearity. Assessment by the hockeystick software derives a threshold value of $773 \mu \mathrm{g} /$ plate for TA1535 but clearly no threshold for YG7104. With the PROAST software the curves were fitted as graphically presented in Fig. 6. The linear fit of induced revertants of YG7104 in the low dose range represents the number of EMS lesions erroneously repaired in absence of ogt alkyltransferase, while the fit of induced revertants for TA1535 represents the number of EMS induced lesions erroneously repaired in presence of the ogt alkyltransferases. It follows that the difference between the two values is representing the number of lesions successfully removed by the ogt system. To visualize the dose dependency of the ogt-repair we plotted in Fig. 7 the ratio of (induced revertants of TA1535)/(induced revertants of YG7104), which can be seen as corresponding to the likelihood of an EMS induced lesion to lead to mutation when ogt repair is active. Erroneous repair appears to approach zero at low concentrations (Fig. 7). On a log-log plot the ratio appears as a straight line with a slope (= exponent) of 1.5 .

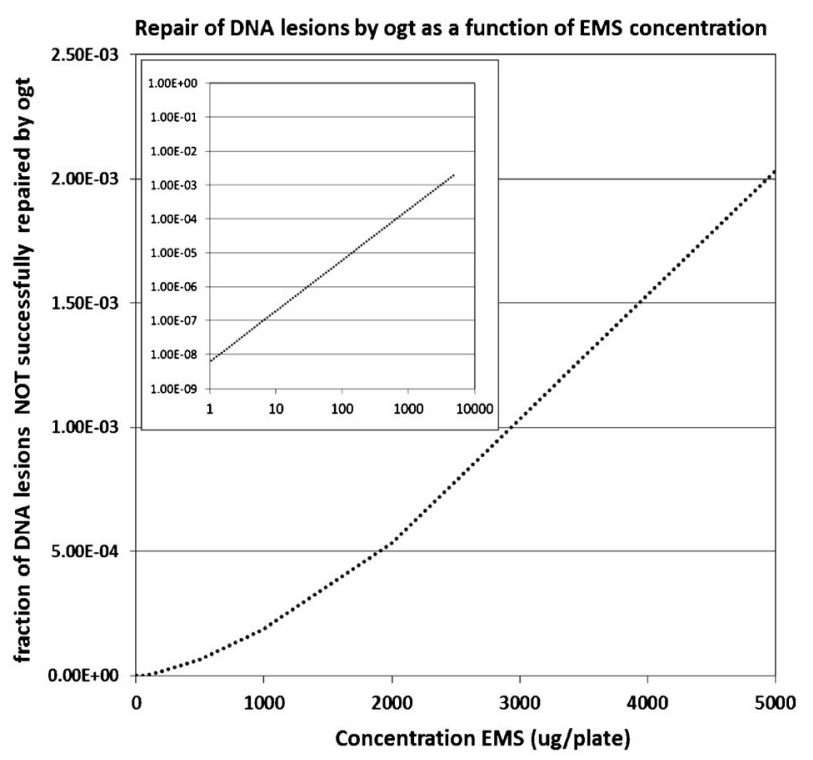

Fig. 7. Graphical presentation of repair by the ogt system. 
Inherently, the exact shape is largely influenced by the curve fitting model and the back-extrapolation to very low concentration levels will carry increasing uncertainty. However, the large numbers of dose levels and plates we have used in the present study increases the confidence of the curve fit. Based on the used model it is concluded that only about 1 lesion in $10^{8}$ is erroneously repaired at $1 \mu \mathrm{g} /$ plate. At the high dose end it can be estimated that still only about 2 in $10^{3}$ lesions are erroneously repaired at $5000 \mu \mathrm{g} /$ plate.

\section{Conclusion}

Emerging evidence from studies using alkylating agents, such as EMS, provide confidence to the conclusion that repair proficient cells have the capacity to eliminate high levels of alkylation damage without apparent increase of genotoxic effects. It is only at doses above the (apparent) threshold that a notable increase of clastogenic and mutagenic effects becomes evident. Our investigation (2) and the study of Doak (1) the dose response of ethylnitrosourea (ENU) did not exhibit a threshold. Since ENU induces ethylation of DNA as does EMS-albeit with a different target specificity-an absence of threshold for ENU appears difficult to rationalize. Therefore it was reassuring that reanalysis of the Doak et al. study $(30,31)$ and further studies focussing on lower dose levels $(3,34)$ provided evidence for thresholds in vivo and in vitro. In contrast, the data on protein and DNA alkylation do not show a thresholded dose response $(2,23,24)$. As has been argued (35), this endpoint can be used to assess the extent of exposure ('biomarker of exposure'), but cannot be used as a 'biomarker of effect' i.e., to estimate the extent of heritable genotoxic consequences. Simple alkylation might be considered as a ubiquitous and easily repairable DNA damage for which error-free repair processes have been evolved early on in the history of life. Other, more complex DNA damages might not be repairable without errors, even at very low doses. Interestingly, however, a recent study on tumour induction in trout (36) exposed to the genotoxin dibenzo[ $a, l]$ pyrene, which induces a host of bulky adducts, has yielded strong evidence for a definitely sub-linear dose response, despite a near linear induction of complex bulky DNA adducts down to very low doses. Similar conclusions have been drawn in a study with aflatoxin as detailed in the presentation by G. Bailey (37). It is evident that linear back extrapolation from TD10 (or TD50) doses to the 'virtual safe' dose (VSD, the dose inducing 1 cancer in a million individuals) yields a value that would be 'too low' by orders of magnitude compared to the estimate by appropriate non-linear curve fitting.

While a general change of the paradigm for risk assessment has to await the accumulation of further data on genotoxins inducing a wide variety of DNA lesions, the current tenet of 'linearity at low doses' for 'mutagenic/clastogenic effects induced by DNA damaging compounds' can be refuted at least for simple alkylating agents.

Acknowledgements: Numerous colleagues are acknowledged for their contributions to the in vivo EMS studies (see references Tox. Letters, vol 190). We thank M. Guerard and A. Zeller for helpful discussions and V. Naessens for excellent technical assistance.

\section{References}

1 Doak SH, Jenkins GJ, Johnson GE, Quick E, Parry EM, Parry JM. Mechanistic influences for mutation induction curves after exposure to DNA-reactive carcinogens. Cancer Res. 2007; 15: 3904-11.

2 Gocke E, Ballantyne M, Whitwell J, Müller L. MNT and MutaTMmouse studies to define the in vivo dose response relations of the genotoxicity of EMS and ENU. Tox Letters. 2009; 190: 286-97.

3 Dobo KL, Fiedler RD, Gunther WC, Thiffeault CJ, Cammerer Z, et al. Defining EMS and ENU dose-response relationships using the Pig-a mutation assay in rats. Mutation Res. 2011; 725: 13-21.

4 Gerber C, Toelle H-G. What happened: The chemistry side of the incident with EMS contamination of Viracept tablets. Tox Letters. 2009; 190: 248-53.

5 Müller L, Singer T. EMS in Viracept-a compilation of events in 2007 and 2008 from the non-clinical point of view. Tox Letters. 2009; 190: 243-7.

6 Lave T, Paehler A, Grimm HP, Birnboeck H. Pharmacokinetic profile of ethyl methanesulfonate in vitro and in animals, including globin ethylation. Tox Letters. 2009; 190: 298-302.

7 Lavé T, Paehler A, Grimm HP, Birnboeck H. Pharmacokinetic modeling of species differences for prediction in humans. Tox Letters. 2009; 190: 303-9.

8 Müller L, Gocke E, Lavé T, Pfister T. Ethyl methanesulfonate toxicity in Viracept-a comprehensive human risk assessment based on threshold data for genotoxicity. Tox Letters. 2009; 190: 317-29.

9 Pfister T, Eichinger-Chapelon A. General 4-week toxicity study with EMS in the rat. Tox Letters. 2009; 190: 271-85.

10 Nohmi T, Yamada M, Masumura K. in vivo approaches to identify mutations and in vitro research to reveal underlying mechanisms of genotoxic thresholds. Genes Environ. 2012; 34: 146-52.

11 Gocke E, Wall M. Statistical assessments for threshold dose response in mice for the induction of micronuclei and $l a c Z$ gene mutation by ethyl methanesulfonate. Tox Letters. 2009; 190: 298-302.

12 Sega GA. A review of the genetic effects of ethyl methanesulfonate. Mutat Res. 1984; 134:113-42.

13 Landini P, Volkert MR. Regulatory responses of the adaptive response to alkylation damage: a simple regulon with complex regulatory features. J Bacteriol. 2000; 182: 6543-9. 
14 Margison GP, Cooper DP, Potter PM. The E. coli ogt gene. Mutat Res. 1990; 233: 15-21.

15 Yamada M, Matsui K, Sofuni T, Nohmi T. New tester strains of Salmonella typhimurium lacking O6-methylguanine DNA methyltransferases and highly sensitive to mutagenic alkylating agents. Mutat Res. 1997; 381: 15-24.

16 Yamada M, Sedgwick B, Sofuni T, Nohmi T. Construction and characterization of mutants of Salmonella typhimurium deficient in DNA repair of O6-methylguanine. J Bacteriol. 1995; 177: 1511-9.

17 Yamada M, Hakura A, Sofuni T, Nohmi T. New method for gene disruption in Salmonella typhimurium: construction and characterization of an ada-deletion derivative of Salmonella typhimurium TA1535. J Bacteriol. 1993; 175: 5539-47.

18 Sedgwick B, Bates PA, Paik J, Jacobs SC, Lindahl T. Repair of alkylated DNA: recent advances. DNA Repair (Amst). 2007; 6: 429-42.

19 Shrivastav N, Li D, Essigmann JM. Chemical biology of mutagenesis and DNA repair: cellular responses to DNA alkylation. Carcinogenesis. 2010; 31: 59-70.

20 Maron DM, Ames BN. Revised methods for the Salmonella mutagenicity test. Mutat Res. 1983; 113: 173-215.

21 Lutz RW, Lutz WK. Statistical model to estimate a threshold dose and its confidence limitsfor the analysis of a sublinear dose response relationship, exemplified for mutagenicity data. Mutation Res. 2009; 678: 118-122.

22 http://www.rivm.nl/en/Library/Scientific/Models/ PROAST (accessed 9 November 2012).

23 Murthy MS, Calleman CJ, Osterman-Golkar S, Segerbäck D, Svensson K. Relationships between ethylation of hemoglobin, ethylation of DNA and administered amount of ethyl methanesulfonate in the mouse. Mutation Res. 1984; 127: 1-8.

24 Matijasević Z, Zeiger E. DNA binding and mutagenicity of ethyl methanesulfonate in wild-type and uvrB cells of Salmonella typhimurium. Mutat Res. 1988; 198: 1-8.

25 Recio L, Osterman-Golkar S, Csanády GA, Turner MJ, Myhr B, Moss O, Bond JA. Determination of mutagenicity in tissues of transgenic mice following exposure to 1,3butadiene and N-ethyl-N-nitrosourea. Toxicol Appl Pharmacol. 1992; 117: 58-64.

26 Gocke E, Müller L, Pfister T, Buergin H. Literature review on the genotoxicity, reproductive toxicity, and car- cinogenicity of ethyl methanesulfonate. Tox Letters. 2009; 190: 254-265.

27 Heddle JA, Martus HJ, Douglas GR. Treatment and sampling protocols for transgenic mutation assays. Environ Mol Mutagen. 2003; 41: 1-6.

28 Gocke E, Müller L. In vivo studies in the mouse to define a threshold fort he genotoxicity of EMS and ENU. Mutation Res. 2009; 678: 101-7.

29 Kaina B, Christmann M, Naumann S, Roos WP. MGMT: key node in the battle against genotoxicity, carcinogenicity and apoptosis induced by alkylating agents. DNA Repair. 2007; 6: 1079-99.

30 Zaïr ZM, Jenkins GJ, Doak SH, Singh R, Brown K, Johnson GE. N-methylpurine DNA glycosylase plays a pivotal role in the threshold response of ethyl methanesulfonate-induced chromosome damage. Toxicol Sci. 2011; 119: 346-58.

31 Johnson GE, Zaïr ZM, Bodger OG, Lewis PD, Rees BJ, Verma JR, Thomas AD, Doak SH, Jenkins GJS. Invetigating mechanisms for non-linear genotoxic responses, and analysing their effects in binary combination. Genes Environ. 2012; 34: 179-85.

32 Jacobs AL, Schär P. DNA glycosylases: in DNA repair and beyond. Chromosoma. 2012; 121: 1-20.

33 Johnson GE, Doak SH, Griffiths SM, Quick EL, Skibinski DO, et al. Non-linear dose-response of DNA-reactive genotoxins: recommendations for data analysis. Mutat Res. 2009; 678: 95-100.

34 Bryce SM, Avlasevich SL, Bemis JC, Phonethepswath S, Dertinger SD. Miniaturized flow cytometric in vitro micronucleus assay represents an efficient tool for comprehensively characterizing genotoxicity dose-response relationships. Mutat Res. 2010; 703: 191-9.

35 Swenberg JA, Fryar-Tita E, Jeong YC, Boysen G, Starr $\mathrm{T}$, et al. Biomarkers in toxicology and risk assessment: informing critical dose-response relationships. Chem Res Toxicol. 2008; 21: 253-65.

36 Bailey GS, Reddy AP, Pereira CB, Harttig U, Baird W, et al. Nonlinear cancer response at ultralow dose: a 40800-animal $\mathrm{ED}(001)$ tumor and biomarker study. Chem Res Toxicol. 2009; 22: 1264-76.

37 Bailey G, Williams D, Orner G, Hendricks J, Pereira C. Cancer risk at ultra-low dose: Lessons learned from 40,000-animal cancer dose-response studies. Genes Environ. 2012; 34: 157-64. 